Research Paper

\title{
Correlation between Peripheral White Blood Cell Counts and Hyperglycemic Emergencies
}

\author{
Wei $\mathrm{Xu}^{1,2},{ }^{*}$, Hai-feng $\mathrm{Wu}^{3}{ }^{*}$, Shao-gang $\mathrm{Ma}^{2},{ }^{*} \bowtie$, Feng $\mathrm{Bai}^{2}$, Wen $\mathrm{Hu}^{2}$, Yue Jin ${ }^{4}$, Hong $\mathrm{Liu}^{2}$ \\ 1. School of Medicine, Southeast University, Nanjing, Jiangsu 210009, China; \\ 2. Department of Endocrinology, the Affiliated Huai'an Hospital of Xuzhou Medical College, Huai'an, Jiangsu 223002, China; \\ 3. Department of Critical Care Medicine, the Affiliated Yixing People's Hospital of Jiangsu University, Yixing, Jiangsu 214200, China; \\ 4. Department of Clinical Laboratory, the Affiliated Huai'an Hospital of Xuzhou Medical College, Huai'an, Jiangsu 223002, China. \\ ${ }^{*}$ Co-first authors. \\ Corresponding author: Shao-gang Ma, Ph.D., M.D. Address: No. 60 South Huaihai Road, Huai'an 223002, China E-mail: \\ mashaogang@163.com Fax/Phone: +86-517-8394 3591.
}

() Ivyspring International Publisher. This is an open-access article distributed under the terms of the Creative Commons License (http://creativecommons.org/ licenses/by-nc-nd/3.0/). Reproduction is permitted for personal, noncommercial use, provided that the article is in whole, unmodified, and properly cited.

Received: 2013.02.26; Accepted: 2013.04.10; Published: 2013.04.18

\begin{abstract}
Objective: To determine the correlation between differential leukocyte counts and hyperglycemic emergencies.

Methods: Fifty patients with diabetic ketoacidosis (DKA), 50 patients with diabetic ketosis (DK), 50 non-DK diabetic patients with stable glycemic control, and 50 normal controls were enrolled. Their total and differential leukocyte counts were measured and evaluated at admission and after treatment.

Results: The patients with DKA and DK had higher plasma glucose levels $(20.84 \pm 6.73 \mathrm{mmol} / \mathrm{L}$, $15.55 \pm 2.6 \mathrm{mmol} / \mathrm{L}$, respectively) and more median leukocytes $\left(13325 / \mathrm{mm}^{3}\right.$ and $6595 / \mathrm{mm}^{3}$, re-

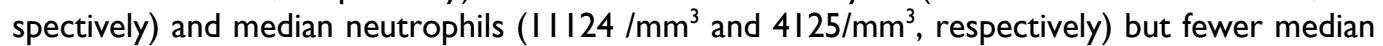
eosinophils $\left(28 / \mathrm{mm}^{3}\right.$ and $72 / \mathrm{mm}^{3}$, respectively) compared to non-DK and control groups (all $p<$ 0.05 ). Acute infection increased the elevating extent. The median leukocyte counts in DK and non-DK patients $\left(6595 / \mathrm{mm}^{3}\right.$ and $6008 / \mathrm{mm}^{3}$, respectively) were within the normal range. The counts of total leukocytes and neutrophils were significantly higher but eosinophils lower in severe DKA cases than in mild/moderate cases $(p<0.05)$. When the DKA and DK and infection resolved, total leukocytes and neutrophils fell, but eosinophils increased. The counts of total leukocytes, neutrophils, and monocytes were negatively correlated with arterial $\mathrm{pH}$ levels $(r=-0.5 \mathrm{I}, r=$ $-0.5 \mathrm{I} 0, r=-0.5 \mathrm{I}$, all $p<0.00 \mathrm{I}$, respectively) and positively correlated with plasma glucose levels $(r=0.722, r=0.733, r=0.632$, all $p<0.05$, respectively) in DKA patients. The arterial $\mathrm{pH}$ level was the most significant factor affecting total leukocytes in DKA $(\beta=0.467, p=0.003)$. The diagnosis analysis showed that higher total leukocyte and neutrophil counts and lower eosinophil counts had a significant ability to reflect the presence of hyperglycemic emergencies.

Conclusion: More total leukocytes and neutrophils but fewer eosinophils was significantly correlated with DKA and DK. Leukocyte counts can add valuable information to reflect the presence of hyperglycemic crisis and acute infection.
\end{abstract}

Key words: Diabetic ketoacidosis, Diabetic ketosis, Leukocytes counts.

\section{Introduction}

Diabetic ketoacidosis (DKA) is a common and serious complication of diabetes mellitus. DKA and diabetic ketosis (DK) have been considered a key clinical feature of type 1 diabetes mellitus (T1DM); 
however, increasing evidence indicates that DKA and DK are also common features of ketosis-prone type 2 diabetes (T2DM) [1]. Most DKA patients require timely treatment. Delays in the diagnosis of DKA can lead to an increase in the risk of advanced complications and 30-day mortality. Patients with DKA are generally poorly controlled prior to their admission, as reflected by high HbA1c levels [2]. Clinical studies have demonstrated non-infectious systemic inflammation in DKA patients, as shown by proinflammatory cytokines $[3,4]$ and increased peripheral white blood cell (WBC) count $[4,5]$.

It is interesting that both acute and chronic diabetic complications are correlated with elevated WBC count. There are studies indicating that an elevated WBC count, even within the normal range, is associated with both macro- and microvascular complications in type 2 diabetes [6]. Higher WBC counts may be associated with the development of retinopathy, albuminuria, and peripheral arterial disease [7-9]. Previous studies have paid much attention to proinflammatory cytokines but little to the routine and reliable WBC count. In addition, the WBC count is routinely detected in diabetic patients but does not receive attention from clinicians.

It should be stressed that previous studies only provided the total leukocyte count without characterizing the differential leukocyte count in hyperglycemic crises $[4,5]$. The influence of DKA and DK on the differential leukocyte count is poorly observed and understood. The high imbalance and releasing characteristics of leukocytes in hyperglycemic emergencies need further study. To our knowledge, there is no available study on the patterns or prognostic value of leukocyte counts in patients with DKA and DK. Accordingly, we aimed to evaluate the changes of total and differential leukocyte counts and the value by reflecting the severity of hyperglycemic emergencies.

\section{Methods}

\section{I Patient recruitment and exclusion criteria}

This study was carried out at the endocrinology and emergency department of two medical college-affiliated hospitals from January 2010 to June 2012 in accordance with the principles of the Declaration of Helsinki (2001). Ethical approval was obtained from the Ethical Committees of Xuzhou Medical College and Jiangsu University. The written informed consent was obtained from all of the participants.

A total of fifty consecutive patients with diabetic ketoacidosis (DKA group), 50 patients with diabetic ketosis (DK group), 50 diabetic patients with a stable condition (non-DK group), and 50 normal control subjects (control group) participated in the study (Table 1). In DKA group included eighteen T1DM and thirty-two ketosis-prone type 2 diabetes patients according to the diagnosis criteria in document concerned [2]. Patients with DKA had a plasma glucose level $>13.90 \mathrm{mmol} / \mathrm{L}$, a urine ketone level defined as moderate to high (+ to +++$)$, and an arterial $\mathrm{pH}$ value $<7.30$ at the time of admission. The criteria for DKA severity were previously developed: mild, $7.20 \leq \mathrm{pH}<$ 7.30; moderate, $7.10 \leq \mathrm{pH}<7.20$; and severe, $\mathrm{pH}<7.10$ $[10,11]$.

The participants underwent a routine medical examination. None of the subjects had heart failure, hematologic disease, or liver or kidney dysfunction, and none of the patients were taking steroids. The DKA, DK, and non-DK patients received medical treatment in the form of nutrition and insulin therapy. Patients with DKA and DK were monitored until ketosis and acidosis resolved.

\subsection{Biochemical assays}

Blood samples were collected into vacutainer tubes from the control and non-DK groups by venipuncture after an overnight fast. In the DKA and DK groups, the blood samples were drawn at admission before the initial therapy and at the time of discharge from the hospital.

Laboratory tests, including routine biochemistry tests and arterial gas analysis, were performed using routine clinical assays in the hospital laboratory. Blood samples were collected for differential WBC counts in tubes with EDTA and immediately processed used a Mindray BC-5500 (Shenzhen, China) automatic blood counting system. The normal reference values for differential WBC counts are the following: total WBCs $4000-10000 / \mathrm{mm}^{3}$, neutrophils $2000-7000 / \mathrm{mm}^{3}$, lymphocytes $800-4000 / \mathrm{mm}^{3}$, monocytes $120-800 / \mathrm{mm}^{3}$, basophils $0-100 / \mathrm{mm}^{3}$, and eosinophils $50-500 / \mathrm{mm}^{3}$.

Glycated hemoglobin A1c (HbA1c) levels were measured using an HbA1c Meter from Bio-Rad Laboratories, Ltd. (Shanghai, China). The HbA1c test result was converted from percent $\mathrm{HbA1c}$ to $\mathrm{HbA} 1 \mathrm{c}$ in $\mathrm{mmol} / \mathrm{mol}$.

\subsection{Statistical methods}

The results were expressed as the mean \pm SD for quantitative variables with normal distributions. The parameters of total and differential WBC counts according to distribution plots and analysis of variance homogeneity were presented as the median and the range (min-max). A pair-wise comparison among the four groups was carried out using the Tukey and Kruskal-Wallis non-parametric ANOVA. MannWhitney $U$ analysis was used for the two subgroups 
nonparametric test. Chi-squared tests were utilized for the comparison of other clinical features. The correlations between the observed variables were analyzed by Pearson's correlation test. The risk markers for the diagnosis of DKA and DK were assessed by multiple logistic analysis. Receiver operating characteristic (ROC) curve analysis was performed to determine the diagnostic performance of the variables. Statistical analyses were conducted with SPSS 18.0 software (SPSS Inc., Chicago, IL) and MedCalc ${ }^{\circledR}$ version 12.1.4.0. A two-tailed $p$ value $<0.05$ was considered statistically significant.

\section{Results}

\section{I Baseline clinical and laboratory characteris- tics}

The baseline demographic and clinical characteristics of the subjects are shown in Table 1. A total of 200 cases were included in the study. There was no difference for the diabetic type. In DKA patients, 28 type 2 diabetics had acute acute respiratory infection. DK and non-DK patients had no infection. There were 22 patients with mild DKA, 13 patients with moderate DKA and 15 patients with severe DKA. The glycemic variables were significantly higher in the three diabetic patient groups (plasma glucose: 20.84 \pm 6.73 $\mathrm{mmol} / \mathrm{L}, 15.55 \pm 2.6 \mathrm{mmol} / \mathrm{L}$, and $9.22 \pm 1.21 \mathrm{mmol} / \mathrm{L}$; and HbA1c: $12.64 \pm 2.95 \%, 11.90 \pm 2.92 \%$, and $8.12 \pm 1.30$, respectively for DKA, DK, and non-DK) than in the control group (plasma glucose: $5.12 \pm 0.21$, HbA1c: $4.53 \pm 0.61 \%$ ) (all $p<0.001$ ). The glycemic variables were higher in the DKA and DK groups than in the non-DK group (all $p<0.05$ ). With respect to age and gender, there were no significant differences among the four groups. The difference in the duration of diabetes did not reach statistical significance. Both the systolic and diastolic blood pressures were lower in the DKA group and higher in the DK group than in the control and non-DK groups $(\mathrm{p}<0.05)$.

\subsection{Total and differential WBC counts}

As shown in Table 2 and 3, this study demonstrated a marked difference in the total and differential WBC counts in the four groups. Of all types of leukocytes, the counts of total WBCs, neutrophils, and eosinophils were significantly altered with glycemic metabolic deterioration. However, the counts of basophils and lymphocytes showed no clear difference. Acute respiratory infection increased the elevating extent of the counts of total WBC and neutrophils but in the counts of lymphocyte basophils and eosinophil reached no difference between infection and non-infection.

Table I. Clinical and biochemical characteristics of the study participants.

\begin{tabular}{|c|c|c|c|c|}
\hline Variable & Control $(n=50)$ & Non-DK $(n=50)$ & DK $(n=50)$ & DKA $(n=50)$ \\
\hline Female/male (n/n) & $26 / 24$ & $25 / 25$ & $26 / 24$ & $24 / 26$ \\
\hline Age (years) & $46.23 \pm 10.12$ & $43.57 \pm 15.82$ & $51.32 \pm 12.10^{\star}$ & $45.05 \pm 13.25$ \\
\hline Diabetic type $(1 / 2)$ & $\mathrm{N} / \mathrm{A}$ & $20 / 30$ & $18 / 32$ & $22 / 28$ \\
\hline Duration of diabetes (years) & $\mathrm{N} / \mathrm{A}$ & $4.45 \pm 1.83$ & $4.55 \pm 2.1$ & $4.65 \pm 2.10$ \\
\hline Systolic blood pressure (mmHg) & $130 \pm 13$ & $138 \pm 14^{*}$ & $145 \pm 21^{\text {** }}$ & $115 \pm 6^{*} \$ 8+t$ \\
\hline Diastolic blood pressure $(\mathrm{mmHg})$ & $83 \pm 5$ & $87 \pm 9$ & $89 \pm 11^{*}$ & $78 \pm 5^{*} 8 s_{+}$ \\
\hline Plasma glucose (mmol/L) & $5.12 \pm 0.21$ & $9.22 \pm 1.21^{* *}$ & $15.55 \pm 2.6^{* \star 8} \S$ & $20.84 \pm 6.73^{* * 8 s \dagger}$ \\
\hline Glycated hemoglobin A1c (\%) & $4.53 \pm 0.61$ & $8.12 \pm 1.30^{* *}$ & $11.90 \pm 2.92^{* * \S}$ & $12.64 \pm 2.95^{* \star \S \S}$ \\
\hline $\mathrm{mmol} / \mathrm{mol}$ & 26 & $65^{* *}$ & $107 \pm 8^{\star \star \S}$ & $115 \pm 9^{* *} \S \S$ \\
\hline Arterial $\mathrm{pH}$ & $\mathrm{N} / \mathrm{A}$ & $\mathrm{N} / \mathrm{A}$ & $7.41 \pm 0.12$ & $7.10 \pm 0.12 \dagger$ \\
\hline Serum $\mathrm{HCO}_{3}(\mathrm{mmol} / \mathrm{L})$ & $\mathrm{N} / \mathrm{A}$ & $\mathrm{N} / \mathrm{A}$ & $19.83 \pm 3.30$ & $9.55 \pm 2.34^{\dagger \dagger}$ \\
\hline
\end{tabular}

Comparison to control group: $\left.\left({ }^{*}\right) p<0.05,{ }^{* *}\right) p<0.001$; comparison to non-DK group: $($ () $p<0.05$, (\$§) $p<0.001$; comparison to DK group: (†) $p<0.05$, (††) $p<$ 0.001 . All values in the table are given as the mean \pm standard deviation

Table 2. The differential white blood cell counts in the study participants at baseline.

\begin{tabular}{|c|c|c|c|c|}
\hline Variable & Control $(n=50)$ & Non-DK $(n=50)$ & DK $(n=50)$ & DKA $(n=50)$ \\
\hline WBCs $\left(\times / \mathrm{mm}^{3}\right)$ & $5550(4001-7710)$ & $6008(4270-7710)^{* *}$ & $6595(3550-13100) * \$$ & $13325(4910-48790)^{* *}$ sst \\
\hline Neutrophils $\left(\times / \mathrm{mm}^{3}\right)$ & 3078 (1800-5005) & $3622(1733-5281)$ & $4125(1233-9503) *$ & $11124(2380-39685)^{* * \$ \&+十 ~}$ \\
\hline Lymphocytes $\left(\times / \mathrm{mm}^{3}\right)$ & 1940 (1087-3814) & $1889(900-2719)$ & $1654(370-3741)^{*}$ & $1515(301-5520)$ \\
\hline Monocytes $\left(\times / \mathrm{mm}^{3}\right)$ & $375(208-652)$ & $344(150-571)$ & $380(29-1044)$ & $608(117-3873)^{* *} \$ s+t$ \\
\hline Basophils $\left(\times / \mathrm{mm}^{3}\right)$ & $6(0-23)$ & $12(0-47)^{\star *}$ & $10(0-41)^{* * \$ 8}$ & $20(0-215){ }^{*} \& 8+t$ \\
\hline Eosinophils $\left(\times / \mathrm{mm}^{3}\right)$ & $109(23-518)$ & 149 (39-443) & $72(0-280)^{*} \mathrm{~s}$ & $28(0-188)^{* * \S \S} \dagger$ \\
\hline
\end{tabular}

Comparison to control group: $\left.\left(^{*}\right) p<0.05,{ }^{* *}\right) p<0.001$; comparison to non-DK group: $($ () $p<0.05$, (\$§) $p<0.001$; comparison to DK group: (†) $p<0.05$, (††) $p<$ 0.001 . All values in the table are given as the median and the range (min-max). 
Table 3. The differential white blood cell counts in DKA patients with and without infection.

\begin{tabular}{llll}
\hline Variable & Non-infection $(\mathbf{n = 2 2})$ & Infection $(\mathbf{n}=\mathbf{2 8})$ & $p$ \\
\hline WBCs $\left(\times / \mathrm{mm}^{3}\right)$ & $10310(4910-22980)$ & $16910(9850-48790)$ & $<0.001$ \\
Neutrophils $\left(\times / \mathrm{mm}^{3}\right)$ & $7560(2380-19030)$ & $15030(8390-39640)$ & $<0.001$ \\
Lymphocytes $\left(\times / \mathrm{mm}^{3}\right)$ & $1470(300-3260)$ & $2206(530-4940)$ & 0.203 \\
Monocytes $\left(\times / \mathrm{mm}^{3}\right)$ & $440(160-1480)$ & $730(320-3870)$ & 0.001 \\
Basophils $\left(\times / \mathrm{mm}^{3}\right)$ & $15(0-50)$ & $20(0-190)$ & 0.290 \\
Eosinophils $\left(\times / \mathrm{mm}^{3}\right)$ & $15(0-520)$ & $20(0-150)$ & 0.851
\end{tabular}

Comparison to non-infection DKA group. All values in the table are given as the median and the range (min-max).

\subsection{Total WBC count}

The median total WBC count increased from the non-DK group to the DKA group. The median WBC count was significantly higher in the DKA group $\left(13325 / \mathrm{mm}^{3}\right)$ than in the other three groups $(p<$ $0.001)$. In the DK and non-DK groups, the median total WBC count was significantly higher $\left(6595 / \mathrm{mm}^{3}\right.$ and $6008 / \mathrm{mm}^{3}$, respectively for DK and non-DK) than the control group $\left(5550 / \mathrm{mm}^{3}\right)($ all $p<0.001)$, although it was still within the normal range.

\subsubsection{Neutrophil count}

The neutrophil count showed similar trends as the total WBC count. The median neutrophil count was significantly higher in the DKA group $\left(11124 / \mathrm{mm}^{3}\right)$ than the DK $\left(4125 / \mathrm{mm}^{3}\right)$ and non-DK $\left(3622 / \mathrm{mm}^{3}\right)$ groups $(p<0.001)$. In the non-DK group, the neutrophil count was lower compared to the DK group $(p<0.05)$. However, there was no difference between the non-DK and control groups $\left(3078 / \mathrm{mm}^{3}\right)$.

\subsubsection{Lymphocyte count}

The lymphocyte count decreased successively from the non-DK group to the DKA group. However, only one significant difference was observed among the four groups. In the DK group, the median lymphocyte count $\left(1654 / \mathrm{mm}^{3}\right)$ was lower compared to the control group $\left(1940 / \mathrm{mm}^{3}\right)(p<0.05)$.

\subsubsection{Monocyte count}

The median monocyte count was significantly higher in the DKA group $\left(608 / \mathrm{mm}^{3}\right)$ than in the other three groups $(p<0.001)$. There was no difference between the DK $\left(380 / \mathrm{mm}^{3}\right)$ and non-DK $\left(344 / \mathrm{mm}^{3}\right)$ and control $\left(375 / \mathrm{mm}^{3}\right)$ groups.

\subsubsection{Basophils and Eosinophil counts}

Both basophils and eosinophil counts were significantly lower in the DKA group than in the other three groups $(p<0.05)$. In the DK group, the median basophils $\left(10 / \mathrm{mm}^{3}\right)$ and the median eosinophil $\left(72 / \mathrm{mm}^{3}\right)$ counts were significantly lower compared with the non-DK $\left(12 / \mathrm{mm}^{3}\right.$ and $149 / \mathrm{mm}^{3}$, respectively for basophils and eosinophil) and control $\left(6 / \mathrm{mm}^{3}\right.$ and $109 / \mathrm{mm}^{3}$, respectively for basophils and eosinophil) groups $(p<0.05)$. The median eosinophil count in the non-DK group showed no difference compared to the control group but the basophils count was higher.

Of all types of leukocytes, the counts of total WBCs, eosinophils, and neutrophils were significantly higher in severe DKA cases compared with mild/moderate cases $(p<0.05)$. However, the mean levels of plasma glucose and HbA1c showed no difference between the mild/moderate cases and severe DKA cases. When DKA and DK resolved after treatment, the levels of $\mathrm{HCO}_{3}$, arterial $\mathrm{pH}$, and plasma glucose returned to normal values. Accordingly, the differential leukocyte counts were significantly decreased to within the normal range in contrast to the levels on admission, except for an increase in eosinophils (all $p<0.05$, data not shown).

\subsection{Correlation and regression analyses}

Bivariate correlation analyses were performed to assess relationships at baseline in DKA and DK patients. In DKA patients, the counts of WBCs, neutrophils, and monocytes were significantly negatively correlated with arterial $\mathrm{pH}$ levels $(r=-0.515, r=$ $-0.510, r=-0.517$, all $p<0.001$, respectively) and positively correlated with plasma glucose levels $(r=$ $0.722, r=0.733, r=0.632$, all $p<0.05$, respectively). However, in DK patients, the total and differential WBC counts were not correlated with the arterial $\mathrm{pH}$ or plasma glucose levels.

The linear regression analyses revealed that arterial $\mathrm{pH}$ was the most significant factor affecting the total WBC count in DKA patients $(\beta=0.467, p=0.003$, 95\% CI: $-45.37-10.28)$. Multiple logistic regression analyses with the significant clinical variables (total WBCs, neutrophils, lymphocytes, monocytes, basophils, and eosinophils) were carried out for the DKA and DK groups respectively. The odds ratios (ORs) and $95 \%$ confidence intervals (CIs) were calculated. The differences in the DKA and DK groups showed no significance. Additionally, the differences in the merged group (DKA and DK) showed no clinical significance $(O R=0.988, p=0.005,95 \%$ CI: 0.980-0.996; 
and $O R=1.001, p=0.015,95 \%$ CI: $1.001-1.002$ for eosinophils and total WBCs, respectively).

\subsection{ROC analyses}

The ROC curve for the total WBC and differential WBC counts for DKA and DK comparison is shown in Figure 1. Accordingly, the AUCs were calculated for evaluation and compared. In DKA patients, the counts of total WBCs, neutrophils, monocytes, and eosinophils showed significant differences but not lymphocyte or basophil counts (Figure 1A). In
DK patients, the eosinophil count showed similar differences in the comparison (Figure 1B).

Because the total WBC and neutrophil counts were significantly higher in both hyperglycemic emergencies but the eosinophil count was significantly lower, to examine the specific ability of the three counts in estimating hyperglycemic crises, the differences in the AUCs of the three counts between DKA and DK were calculated, and significant differences were observed ( all $p<0.05$ ) (Figure 2).
A

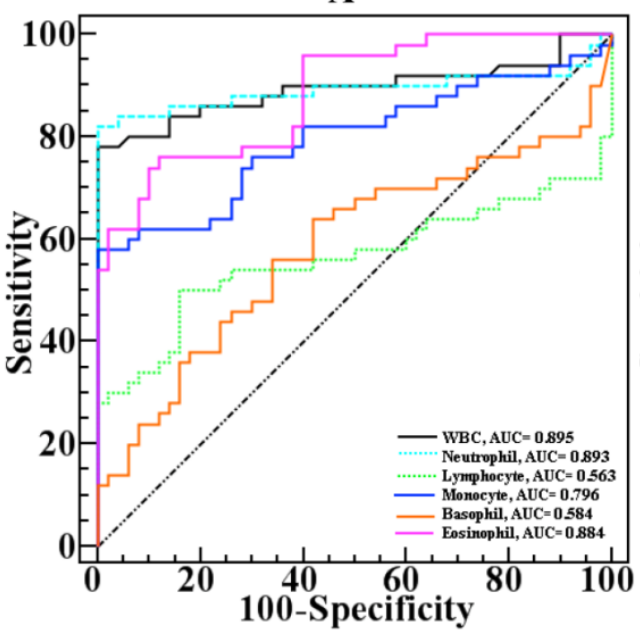

B

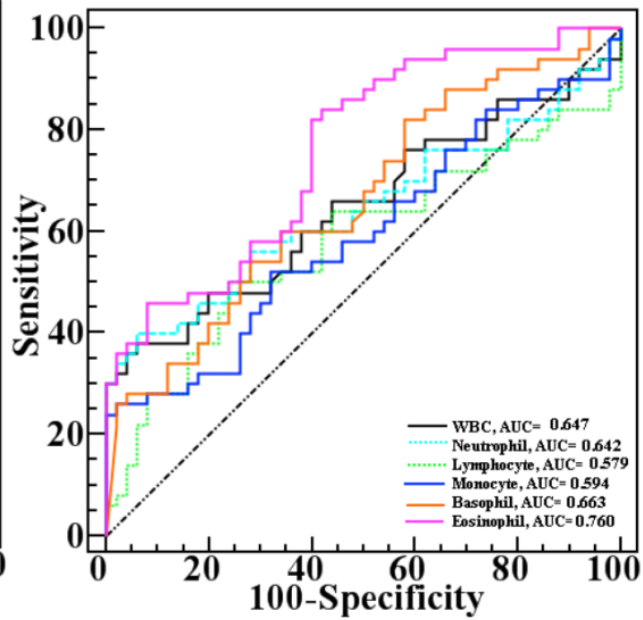

\begin{tabular}{|c|c|c|c|c|c|c|}
\hline \multirow{2}{*}{$\begin{array}{l}\text { Variable } \\
\text { Fig A }\end{array}$} & \multirow[t]{2}{*}{ AUC } & \multirow[t]{2}{*}{ S.E. } & \multirow[t]{2}{*}{$95 \% \mathrm{CI}$} & \multirow[t]{2}{*}{ Cut-off } & \multicolumn{2}{|c|}{ Sensitivity Specificity } \\
\hline & & & & & $(\%)$ & $\%)$ \\
\hline WBCs & 0.895 & 0.037 & $0.817-0.947$ & $>7710$ & 78.0 & 100.0 \\
\hline Neutrophils & 0.893 & 0.040 & $0.815-0.946$ & $>5281$ & 82.0 & 100.0 \\
\hline Lymphocytes & 0.563 & 0.063 & $0.460-0.662$ & $\leq 1462$ & 50.0 & 84.0 \\
\hline Monocytes & 0.796 & 0.047 & $0.703-0.870$ & $>571$ & 58.0 & 100.0 \\
\hline Basophils & 0.584 & 0.060 & $0.481-0.682$ & $>19$ & 56.0 & 66.0 \\
\hline Eosinophils & 0.884 & 0.032 & $0.805-0.939$ & $\leq 58$ & 74.0 & 90.0 \\
\hline Variable & AUC & S.E. & $95 \% \mathrm{CI}$ & Cut-off & Sensitivity & pecificity \\
\hline Fig B & & & & & $(\%)$ & $(\%)$ \\
\hline WBCs & 0.647 & 0.056 & $0.545-0.740$ & $>7480$ & 38.0 & 94.0 \\
\hline Neutrophils & 0.642 & 0.057 & $0.540-0.735$ & $>4652$ & 40.0 & 94.0 \\
\hline Lymphocytes & 0.579 & 0.059 & $0.476-0.677$ & $\leq 1609$ & 50.0 & 76.0 \\
\hline Monocytes & 0.594 & 0.058 & $0.491-0.691$ & $>522$ & 26.0 & 98.0 \\
\hline Basophils & 0.663 & 0.054 & $0.561-0.754$ & $\leq 10$ & 54.0 & 72.0 \\
\hline Eosinophils & 0.760 & 0.047 & $0.664-0.840$ & $\leq 132$ & 82.0 & 60.0 \\
\hline
\end{tabular}




\begin{tabular}{lllll}
\hline \multicolumn{1}{c}{ Variable } & $\begin{array}{l}\text { Z statistic } \\
\text { Fig A }\end{array}$ & $\begin{array}{c}p \\
\text { Fig A }\end{array}$ & $\begin{array}{l}\text { Z statistic } \\
\text { Fig B }\end{array}$ & $\begin{array}{c}p \\
\text { Fig B }\end{array}$ \\
\hline Total WBC vs Neutrophils & 0.098 & 0.922 & 0.160 & 0.873 \\
Total WBC vs Lymphocytes & 4.503 & $<0.001$ & 0.725 & 0.468 \\
Total WBC vs Monocytes & 2.992 & 0.003 & 0.842 & 0.400 \\
Total WBC vs Basophils & 4.757 & $<0.001$ & 0.189 & 0.850 \\
Total WBC vs Eosinophils & 0.241 & 0.810 & 1.466 & 0.142 \\
Neutrophils vs Lymphocytes & 4.856 & $<0.001$ & 0.805 & 0.421 \\
Neutrophils vs Monocytes & 2.500 & 0.012 & 0.660 & 0.510 \\
Neutrophils vs Basophils & 4.423 & $<0.001$ & 0.266 & 0.790 \\
Neutrophils vs Eosinophils & 0.187 & 0.852 & 1.668 & 0.095 \\
Lymphocytes vs Monocytes & 2.942 & 0.003 & 0.160 & 0.873 \\
Lymphocytes vs Basophils & 0.218 & 0.827 & 1.107 & 0.268 \\
Lymphocytes vs Eosinophils & 5.041 & $<0.001$ & 3.003 & 0.003 \\
Monocytes vs Basophils & 3.169 & 0.002 & 0.798 & 0.425 \\
Monocytes vs Eosinophils & 1.528 & 0.127 & 2.010 & 0.044 \\
Eosinophils vs Basophils & 4.210 & $<0.001$ & 1.629 & 0.103 \\
\hline
\end{tabular}

Figure I. Comparison of the ROC plots of total and differential WBC counts with respect to their ability to reflect significant differences in the AUCs for DKA $(\mathbf{A})$ and DK $(\mathbf{B})$.
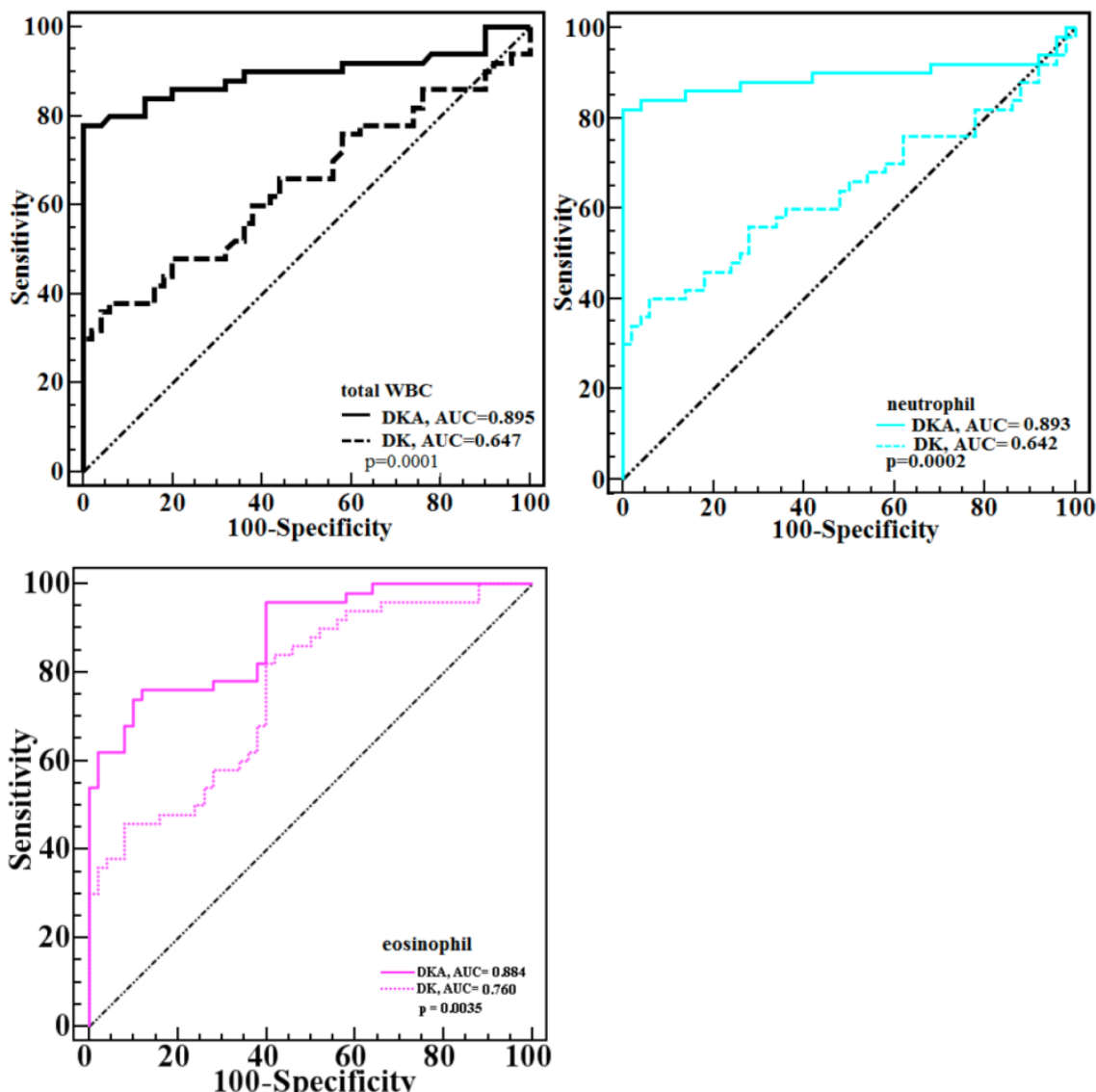

Figure 2. Comparison of the ROC plots of total WBC, neutrophil, and eosinophil counts between DKA and DK patients. Significant differences were found ( $p<0.00 \mathrm{I}, p<0.00 \mathrm{I}, p=0.004$, respectively for total WBC, neutrphil, and eosinophil). 


\section{Discussion}

The present study demonstrated that the total and differential leukocyte counts were significantly altered in patients with hyperglycemia from a stable condition to ketoacidosis. Acute infection increased the elevating extent of the counts of total WBC and neutrophils. Patients with ketoacidosis and ketosis had higher counts of total WBCs and neutrophils but lower counts of eosinophils compared to non-ketosis diabetic patients and control subjects. Though basophils and eosinophil counts make up a very small proportion of total WBC count, receiver operating characteristic curves showed that the three types of leukocytes (total WBCs, neutrophils, and eosinophils) can equally reflect the presence of diabetic ketoacidosis. To our knowledge, this study dealed with a theme that the existing literature data are limited.

Hyperketonemia with acidosis has been considered an acute complication of poorly controlled or newly diagnosed diabetes mellitus. The degree of DKA severity is significantly associated with the arterial $\mathrm{pH}$ level. Higher $\mathrm{H}^{+}$levels may cause leukocytes to be produced and released. In the present study, of all types of leukocytes, the counts of total WBCs, eosinophils, and neutrophils were significantly different between the severe ketoacidosis cases and the mild/moderate cases. The total and differential leukocyte counts were significantly elevated in the ketoacidosis group compared with patients without acidosis; however, the eosinophil count was significantly lower in both hyperglycemic emergencies. The elevated levels of ketone bodies can result in oxidative damage, accelerated apoptosis, and the inhibition of cell growth in monocytes, which can lower the monocyte count [12]. Ketones may have a similar effect on the eosinophil count. Logistic regression and ROC analysis showed that lower eosinophil counts may be a more suitable risk marker than lymphocyte, monocyte, or basophil count for the diagnosis of ketoacidosis.

It has been noted that diabetic ketoacidosis is associated with an elevated level of active systemic inflammatory processes and oxidative stress [3-5]. In addition, hyperketonemia increases monocyte adhesion to endothelial cells and IL-8 and MCP-1 secretion in monocytes [13]. Extracellular acidosis downregulates most of the

Hemostatic platelet functions and promotes those involved in amplifying the neutrophil-mediated inflammatory response [14]. The activation of a subgroup of T-lymphocytes leads to the increased production of reactive oxygen species, cytokines, and growth factor receptors [15]. Based on previous stud- ies and our results, it can be concluded that these proinflammatory cytokines, reactive oxygen species, and adhesion molecules can derive from changes in leukocytes. The inflammation in the diabetic ketoacidosis state is associated with the immune response in polymorphonuclear cells. Inflammation, as indicated by elevated total WBC and neutrophil counts, may play a role in the development of profound pathophysiology.

Automated measurement of the leukocyte indices has many advantages, such as a lower price and more convenient application from a technical perspective. Clinicians should pay more attention to leukocyte spectrum when diabetic patients develop significant hyperglycemia. Previous studies showed that total WBC, neutrophil, lymphocyte, monocyte and eosinophil counts but not basophil count were increased in T2DM patients with metabolic syndrome. In addition, the elevated leukocyte counts were within the normal range [6-9, 16]. In our study, the median eosinophil count was $149 / \mathrm{mm}^{3}$ in non-ketosis patients and $109 / \mathrm{mm}^{3}$ in control subjects. The former value was high but showed no significant difference. The neutrophil count of non-ketosis patients was higher than in controls, although it was within the normal range. Leukocyte activation is likely to contribute to the known increased risk of diabetic complications. Both the differences between the study groups and the correlation were showed. To determine which type cell count has significance, logistic regression and ROC analysis were used. ROC analysis has the the advantage for refecting the presense. And ROC analysis curve intuitively showed the value of differential cell counts, especial eosinophil count.

Hyperglycemic crises are characterized by varying degrees of the depletion of water and electrolytes but increased cortisol [5]. In this study, patients with ketoacidosis had lower blood pressure. The success of the treatment is significantly correlated to the correct management of rehydration, metabolic acidosis, and electrolyte replacement [17]. Treatment with insulin had a favorable effect on the patient's glycemic control and corrected differential leukocyte counts. These findings suggest that diabetic ketoacidosis and ketosis may promote the wide range of pathophysiologic adaptations and changes. All these conditions are commonly found in hyperglycemic crises patients. The alterations found in this study may be useful to help to understand, at least in part, the pathophysiology of diabetic hyperketonemia. A limitation of our study is that the results represent preliminary evidence from a small cross-sectional study. We did not show a relationship between the differential leukocyte 
counts and ketoacidosis complications, such as cerebral edema, pulmonary edema, and abdominal pain. It is know that DKA has already well-established diagnostic criterion. The demargination of neutrophils as a stress response is a frequent cause of elevated neutrophils in acute medical emergencies. Leukocytosis is a common observation in acute illness and the value of utilizing this phenomenon in diagnosing hyperglycemic crises is very limited. To a certain degree, elevated peripheral white blood cell counts can reflect DKA presense. We designed this study to detect the ability for refecting the presense of DKA. Subclinical atherosclerosis is correlated positively with fasting glucose levels and negatively with units of insulin $/ \mathrm{kg} /$ day administered in T1DM individuals. In addition, blood cell counts could be a putative marker for estimating both acute complication and subclinical atherosclerosis [18]. Though our results cannot replace traditional diagnostic criterion, this study add complementray laboratory data and the patterns of total and differential leukocyte counts.

Taken together, this study demonstrated the significant alteration of total and differential leukocyte counts in diabetic patients with hyperglycemic crises. It was determined that higher counts of total WBCs and neutrophils, especially lower counts of eosinophils, should be interpreted with caution in relation to hyperglycemic emergencies.

\section{Acknowledgments}

We acknowledge and thank all participants for their cooperation and sample contributions.

\section{Competing Interests}

The authors have no competing interests to declare. Author contributions: M. S. G. and W. H. F. designed and conducted the study. M. S. G. and X. W. wrote the manuscript. B. F., X. W., H. W., L. H., W. H. F., and J. Y. collected and analyzed the data.

\section{References}

1. Smiley D, Chandra P, Umpierrez GE. Update on diagnosis, pathogenesis and management of ketosis-prone Type 2 diabetes mellitus. Diabetes Manag (Lond). 2011; 1: 589-600.

2. Barski L, Nevzorov R, Rabaev E, et al. Diabetic ketoacidosis: clinical characteristics, precipitating factors and outcomes of care. Isr Med Assoc J. 2012; 14: 299-303.

3. Ma SG, Jin $Y, X u$ W, et al. Increased serum levels of ischemia-modified albumin and C-reactive protein in type 1 diabetes patients with ketoacidosis. Endocrine. 2012; 42: 570-6.

4. Stentz FB, Umpierrez GE, Cuervo R, et al. Proinflammatory cytokines, markers of cardiovascular risks, oxidative stress, and lipid peroxidation in patients with hyperglycemic crises. Diabetes. 2004; 53: 2079-86.

5. Karavanaki K, Karanika E, Georga S, et al. Cytokine response to diabetic ketoacidosis (DKA) in children with type 1 diabetes (T1DM). Endocr J. 2011; 58: 1045-53.

6. Tong PC, Lee KF, So WY, et al. White blood cell count is associated with macro- and microvascular complications in chinese patients with type 2 diabetes. Diabetes Care. 2004; 27: 216-22.
7. Moradi S, Kerman SR, Rohani F, et al. Association between diabetes complications and leukocyte counts in Iranian patients. J Inflamm Res. 2012; 5: 7-11.

8. Woo SJ, Ahn SJ, Ahn J, et al. Elevated systemic neutrophil count in diabetic retinopathy and diabetes: a hospital-based cross-sectional study of 30,793 Korean subjects. Invest Ophthalmol Vis Sci. 2011; 52: 7697-703

9. Papazafiropoulou A, Kardara M, Sotiropoulos A, et al. Plasma glucose levels and white blood cell count are related with ankle brachial index in type 2 diabetic subjects. Hellenic J Cardiol. 2010; 51: 402-6.

10. Rosenbloom AL. The management of diabetic ketoacidosis in children. Diabetes Ther. 2010; 1: 103-20.

11. Wolfsdorf J, Craig ME, Daneman D, et al. International Society for Pediatric and Adolescent Diabetes. Diabetic ketoacidosis. Pediatr Diabetes. 2007; 8: 28-43.

12. Jain SK, Kannan K, McVie R. Effect of hyperketonemia on blood monocytes in type-I diabetic patients and apoptosis in cultured U937 monocytes. Antioxid Redox Signal. 1999; 1: 211-20.

13. Rains JL, Jain SK. Hyperketonemia increases monocyte adhesion to endothelial cells and is mediated by LFA-1 expression in monocytes and ICAM-1 expression in endothelial cells. Am J Physiol Endocrinol Metab. 2011; 301: E298-306.

14. Etulain J, Negrotto S, Carestia A, et al. Acidosis downregulates platelet haemostatic functions and promotes neutrophil proinflammatory responses mediated by platelets. Thromb Haemost. 2012; 107: 99-110.

15. Kitabchi AE, Stentz FB, Umpierrez GE. Diabetic ketoacidosis induces in vivo activation of human T-lymphocytes. Biochem Biophys Res Commun. 2004; 315:404-7.

16. Shim WS, Kim HJ, Kang ES, et al. The association of total and differential white blood cell count with metabolic syndrome in type 2 diabetic patients. Diabetes Res Clin Pract. 2006; 73: 284-91.

17. Rosenbloom AL. The management of diabetic ketoacidosis in children. Diabetes Ther. 2010; 1: 103-20.

18. Faienza MF, Acquafredda A, Tesse R, et al. Risk factors for subclinical atherosclerosis in diabetic and obese children. Int J Med Sci. 2013; 10: 338-43. 